
$\angle$ Research Square
Preprints are preliminary reports that have not undergone peer review.
They should not be considered conclusive, used to inform clinical practice,
or referenced by the media as validated information.

\title{
Gene modules and non-coding RNAs involved in pancreatic tumorigenesis through acinar ductal metaplasia
}

\author{
Huairong Zhang \\ Shanghai General Hospital \\ Bingyin Shi \\ Xi'an Jiaotong University \\ ZU-HUA GAO \\ McGill University Health Centre \\ BO GAO ( $\square$ pkuph_bogao@163.com) \\ Peking university people's hospital
}

\section{Research article}

Keywords: pancreatic adenocarcinoma, acinar to ductal metaplasia, pancreatic intraepithelial neoplasia, gene modules, non-coding RNAs

Posted Date: November 20th, 2020

DOI: https://doi.org/10.21203/rs.3.rs-31936/v2

License: (9) (1) This work is licensed under a Creative Commons Attribution 4.0 International License. Read Full License 


\section{Abstract}

Background: Acinar ductal metaplasia (ADM) is a recently identified precursor lesion that can progress through pancreatic ductal intraepithelial neoplasia (PanIN) to pancreatic ductal adenocarcinoma (PDAC). However, the genetic alterations and the transcriptional regulators at work during the process of ADMdriven PDAC tumorigenesis are largely unknown. We applied a multidimensional integration strategy to unveil the gene modules and non-coding RNAs that drive the ADM-PanIN-PDAC process.

Methods: GSE40895 and the microarray datasets were integrated to unmask the regulators linked to ADM, PanIN and PDAC. Based on the differentially expressed genes and protein-protein interaction (PPI) networks for each stage, overlapping and crosstalk gene modules in ADM-PanIN-PDAC were identified using the search tool for the retrieval of interacting genes (STRING) and Cytoscape. The functions of these modules were elucidated by gene ontology (GO) analysis. The expression levels of hub genes and survival analysis were investigated in human PDAC via gene expression profiling interactive analysis (GEPIA). The MiRDB database was used to predict potential non-coding RNAs (ncRNAs) capable of regulating overlap and crosstalk genes.

Results: We found several bridging ADM gene modules (e.g. SMARCA1 and H2AFZ), PanIN gene modules (e.g. HDAC11 and SMARCA2) and PDAC gene modules (e.g. OLFR239 and CLIP3). They were enriched in nucleosome assembly, chromatin organization and G-protein coupled receptor signalling pathways by GO analysis. MicroRNAs (e.g. mmu-miR-335-5p and mmu-miR-669n) and IncRNAs (e.g. H19 and Gm14207) took part in this ample crosstalk by regulating the gene expression.

Conclusions: SMARCA1, SMARCA2 and CLIP3 were identified as novel crosstalk genes and potential prognostic biomarkers for ADM-driven PDAC carcinogenesis. After validation in clinical and functional studies, transcriptional regulatory non-coding RNAs targeting crosstalk and overlapping genes could represent effective targets for early PDAC intervention.

\section{Background}

Pancreatic ductal adenocarcinoma (PDAC) is the fourth leading cause of cancer death in both men and women[1, 2]. Poor survival is attributed to multiple factors including an incomplete understanding of PDAC pathogenesis, the lack of sensitive and specific biomarkers for early diagnosis, and because it is refractory to most treatment regimens[3]. Chronic pancreatitis has been documented as a predisposing factor for pancreatic cancer[4, 5]. Acinar-ductal metaplasia (ADM) often results from pancreatitis[6] and is also frequently associated with pancreatic intraepithelial neoplasia (PanIN)[7], making it a possible link between inflammation and malignancy. ADM is characterized by the loss of morphology and genetic signatures of acinar cells and the gain of characteristics of pancreatic ducts[8, 9]. Lineage tracing experiments demonstrated that PanIN lesions arise from ADM and could eventually progress to PDAC[10]. Therefore, it is conceivable to expect that ADM, PanIN and PDAC may have common genetic alterations and signaling pathway aberrations [11].

The activation of oncogenes (KRAS, BRAF, AKT2, GATA6 and MYC), inactivation of tumor suppressor genes (CDKN2A, TP53, SMAD4/DPC4 and BRCA2) and mutations in genome maintenance and repair genes (MLL, ATM and ARID1A) have all been implicated in PDAC carcinogenesis[12, 13]. The most common mutation in PDAC, KRAS, triggers its downstream signaling cascades, such as the mitogen-activated protein kinase (MAPK), phosphoinositol-3 kinase (PI3K), and transforming growth factor- $\beta$ (TGF- $\beta$ ) signaling pathways, to regulate cell differentiation, proliferation and survival[14]. Epigenetic processes that comprise DNA-methylation, post-transcriptional modification of histones and remodeling of the nucleosome can regulate gene expression without changing the DNA sequence, and eventually contribute to PDAC pathogenesis[15]. MicroRNAs (miRNAs such as miR-21, miR-217) and long noncoding RNAs (IncRNAs such as H19) also play important roles in PDAC carcinogenesis, invasion and metastasis[16]. However, all of these genetic and epigenetic alterations in PDAC were discovered in isolation, and the crosstalk between genetic alterations involved in the ADM-PanIN-PDAC three-stage progression model and the interaction of this network with epigenetic alterations (miRNAs and IncRNAs) remain ill-defined. In this study using STRING and Cytoscape, we identified potential driver genes promoting the development of ADM-PanIN-PDAC and determined the miRNAs and IncRNAs regulating the expression of genes involved in this threestage progression model. Characterization of the genetic and epigenetic alterations during ADM-PanIN-PDAC progression may lead to the identification of molecular signatures for PDAC carcinogenesis that could be utilized clinically for disease prevention, early diagnosis, and treatment.

\section{Methods}

\section{Selection of samples and differentially expressed genes.}

We downloaded GSE40895 from the Gene Expression Omnibus containing whole genome information on ADM, PanIN and PDAC through the National Center of Biotechnology Information. The ADM data module was derived from an experimental pancreatitis model, where the ADM were enriched at day 1 to Day 3 after caerulein injection [17]. Complete regeneration and repair was observed 5 days after insult in the wild type mouse. Pdx1-Cre;LSLKrasG12D/+ mice aged 10 and 20 wk (termed KrasG12D) that harbor PanIN lesions and a subset that develop PDAC were applied as PanIN and PDAC groups. The formation of ADM, PanIN and PDAC were validated primarily by histopathology followed by Dolichos biflorus Agglutinin (DBA) lectin immunohistochemical staining, RNA expression level of certain ductal and acinar markers, and gene expression profiling [18].Gene expression was standardized using the Limma package for $\mathrm{R}$ (version 3.8.6)[19]. P values less than 0.05 were considered as statistically significant. Heatmaps of DEGs were drawn in R.

\section{Establishing PPI networks and gene modules}

To understand the interactions among ADM, PanIN and PDAC, protein-protein interaction (PPI) was obtained from the STRING database and its network was analyzed with the Limma package for R. Based on the PPI network, we used the MCODE plugin in Cytoscape (http://apps.cytoscape.org/apps/mcode) to select gene modules from ADM, PanIN and PDAC, respectively.

\section{Overlapping gene modules in ADM, PanIN and PDAC}




$$
\mathrm{p}=1-\sum_{i=0}^{m-1} \frac{\left(\begin{array}{l}
n \\
i
\end{array}\right)\left(\begin{array}{l}
N-n \\
M-i
\end{array}\right)}{\left(\begin{array}{l}
N \\
M
\end{array}\right)}
$$

hypergeometric test with the following formula[20]: $\mathrm{M}, \mathrm{n}, \mathrm{N}$ and $\mathrm{m}$ represent the total number of genes in the ADM module, PanIN module, STRING database, and overlap modules, respectively. Overlapping gene modules between samples of PDAC and PanIN were also selected by the formula mentioned above. In this case $\mathrm{M}$ and $\mathrm{n}$ represent the number of genes in PanIN and PDAC, respectively, while the other values remain the same as above. A P value less than 0.05 was considered statistically significant. The same test was run again on these two overlapping gene modules. We finally obtained the significant overlapping gene modules of ADM-PanIN-PDAC. Cytoscape was used to visualize the interaction network of the overlapping gene modules.

\section{Crosstalk gene modules in ADM, PanIN and PDAC}

Whether the crosstalk of two gene modules is statistically significant depends on the number of interactions between two certain PPI networks and random computation[20]. One pair of sub-network modules of ADM and PanIN (or PanIN and PDAC) had $m$ times of participation interaction in actual conditions. The original PPI network was randomized 1000 times by maintaining the degree of distribution of the unchanged nodes. The two sub-network modules with the same size as the original network modules were randomly screened. We computed the interaction times in random sub-network modules in the same pair of ADM and PanIN (or PanIN and PDAC) modules. The $p$ value for the significance of the interaction between a single pair of sub-network modules was calculated as the randomized simulation computation of interaction times larger than the actual participation interaction times divided by 1000 times. Interactive sub-network modules with a $\mathrm{p}$ value less than 0.05 were considered to be significant interactive sub-networks.

\section{Gene ontology analysis of overlapping and crosstalk gene modules}

The function of significant overlapping and crosstalk gene modules was investigated by GO analysis through the database for annotation, visualization and integrated discovery (DAVID) (https://david.ncifcrf.gov). Fisher's exact test was applied and a P value less than 0.05 was defined as statistically significant.

\section{MicroRNA and IncRNA network regulating the overlapping and crosstalk gene modules}

The MiRDB database was used to predict those microRNAs capable of regulating significant overlapping and crosstalk gene modules with the bioinformatic tool MirTarget. All of the mouse IncRNA information was obtained from deepbase (http://biocenter.sysu.edu.cn/deepBase/browser.php). Co-expression of IncRNA and potential target genes was acquired based on Pearson correlation by R. Only those with both a P value less than 0.05 and a correlation value more than 0.7 were considered as predicted IncRNA.

\section{Differentially expressed and potentially prognostic overlapping or crosstalk genes in human PDAC}

To explore the expression level of overlapping and crosstalk genes in human PDAC samples, we used the GEPIA tool[21] to perform differential expression analysis in 171 normal pancreatic tissue samples and 179 PDAC tissue samples produced by The Cancer Genome Atlas (TCGA) and Genotype-Tissue Expression (GTEx) projects. Genes with absolute $\log _{2}$ Fold Change $\left(\left|\log _{2} \mathrm{FC}\right|\right)>1$

and $P<0.05$ are considered to be differentially expressed. Overall survival analysis was also performed by the GEPIA tool. It classified the high-expression and low-expression cohorts according to the median for log-rank test (Mantel-Cox test). Hazard ratios (HR) and confidence intervals (Cl) were given. Genes with $P$ $<0.05$ were considered to be significant potential prognostic biomarkers for PDAC. Results with statistical significance were reported.

\section{Results}

\section{DEGs and PPI networks in ADM-PanIN-PDAC}

In the GSE40895 dataset, a total of 1658 DEGs in ADM (972 upregulated, 686 downregulated), 557 DEGs in PanIN (254 upregulated, 303 downregulated) and 705 DEGs in PDAC (355 upregulated, 350 downregulated) were identified among samples compared with matched non-precursor lesions or non-tumor tissue samples, respectively. A heatmap of the DEG expression was also created in the corresponding ADM, PanIN and PDAC groups (Figure S1). The names of the genes used for the heatmap are presented in Tables S1-S3. To investigate the regulatory correlations of the DEGs in ADM, PanIN and PDAC, a PPI network of the DEGs was mined. 935 DEGs from a total of 1658 DEGs in ADM (Fig. 1) were filtered into the DEG PPI network complex, including 6278 edges and 935 nodes. The degree value of the genes was indicated by the size of the nodes. The top 10 largest nodes in ADM included Ehmt1, Hdac3, Cecr2, Cdk6, Ranbp2, Bptf, Actg1, Acta1, Ncbp1 and Cul3. We then clarified 639 PPI pairs among 261 filtered DEGs in PanIN and found 10 nodes with a higher degree (Rhob, Hist1h3g, Hist1h4j, Hist4h4, Hdac11 and Cdk17, Fig. 1c). Among all the 705 DEGs in PDAC, 320 DEGs were filtered into the PPI network with 320 nodes and 777 edges. Rhoq, Actr8, Frk, Zap70, Mras, Akt3, Lyn, Rab25, Pik3c3 and Jup were the top 10 largest nodes in the PPI network of PDAC.

\section{Significant overlapping gene modules in ADM-PanIN-PDAC}

In total, twenty-six, seven and nineteen gene modules were selected from the PPI networks of ADM, PanIN and PDAC, respectively, based on the MCODE plugin of Cytoscape software. Using hypergeometric tests of the genes above, we found one pair of modules containing 4 significant overlapping genes between ADM module-4 and PanIN module-1 (Fig. 2, $P=0.012$,). Detailed statistical information on overlapping genes is shown in Table S4. DEGs in ADM module-4 
were enriched in nucleosome assembly, positive epigenetic regulation and initiation of DNA template transcription by GO analysis. Interestingly, DEGs in PanIN module-1 were enriched in similar functions including epigenetic regulation of gene expression, DNA-template transcription, and DNA methylation. 75\% of all the genes annotated in both ADM and PanIN modules were members of the histone family, strongly indicating its potential role in promoting ADM to PanIN. Coincidently, all of the 4 overlapping genes (Hist1h2an, Hist1h4c, Hist1h4m and Hist4h4) belong to the core histone family, H2A and H4, suggesting that they may play critical roles in promoting ADM to PanIN. To further understand the major functions of the overlapping genes, we performed a GO analysis based on the DAVID database (Table 1). No significant overlapping genes were found between PanIN and PDAC modules. This result indicates that overlapping genes are highly involved in the initial stages of PDAC (ADM and PanIN), rather than the PanIN-PDAC stage. However, whether overlapping genes exist between PanIN-PDAC based on other databases remains to be explored. Therefore, the mechanisms for regulating ADM-PanIN-PDAC progression may rely mostly on crosstalk, especially at the PanIN-PDAC stage.

\section{Crosstalk gene modules in ADM-PanIN-PDAC.}

\section{Crosstalk modules between ADM and PanIN}

Three pairs of PPI sub-networks between ADM and PanIN with significant crosstalk modules were found. There were 63,35 and 20 crosstalk pairs between ADM module-1 and PanIN module-4 (Fig. 3A, $P=0.012$ ), ADM module-4 and PanIN module-4 (Fig. 3B, $P<0.0001$ ), ADM module-5 and PanIN module-6 respectively (Fig. $3 \mathrm{C}, P<0.0001$ ). Consistent with the overlapping gene modules between ADM and PanIN, members of histone family account for the majority of crosstalk genes. Hist1h3g and HDAC11 in PanIN module-4 had crosstalk with not only HDAC3, Hist1h4h, Hist1h2ad and Hist1h2bn in ADM module-1 and 4, but also with HDAC6 in PDAC module-9 (Fig. 3F, $P=0.002$ ). Notably, all the crosstalk genes between ADM module-5 and PanIN module-6 ( $P<0.0001)$ were from the olfactory receptor (OR/OLFR) superfamily.

\section{Crosstalk modules between PanIN and PDAC}

Seven crosstalk modules between PanIN and PDAC were uncovered. PanIN module-4 cross talks with three different PDAC modules, indicating the close interaction between these two stages (Fig.3D-3F, $P=0.014, P=0.040, P=0.002$ respectively). The OR family also links PanIN module- 6 and PDAC module1(Fig.3G, $P=0.003$ ). ORs are involved in cell differentiation and carcinogenesis[22]. However, their roles in the pancreas are unclear. PanIN module-3 interacted with both PDAC module-13 (Fig.3H, $P<0.001$ ) and -10 (Fig.3J, $P<0.0001$ ). An interaction between PanIN module-1 and PDAC module-9 (Fig.3I, $P=0.036$ ) was also uncovered. These crosstalk modules may provide new insights into how PanIN gradually progresses to PDAC.

\section{Crosstalk modules in ADM-PanIN-ADM}

We finally combined all the 10 crosstalk modules above to select crosstalk modules among the three different modules (ADM, PanIN and PDAC). Two crosstalk sub-networks of ADM-PanIN-PDAC were discovered (Fig.3K and Fig.3L). Detailed statistical information on the combined crosstalk genes are shown in Table S6. Interestingly, most genes involved in the crosstalk of ADM-PanIN-PDAC are from the histone family. Mutation and modification of histones may promote ADM progression to PDAC through multiple pathways including nucleosome assembly, microtubule-based processes, and chromatin modification, as predicted by GO analysis (Table 2). The top 10 genes regulating ADM-PanIN-PDAC with the highest degree were Chd1, Bptf, Smarca1, Cecr2『H2afz in ADM module-1, Smarca2 in PanIN module-4, and Frk, Clip3, Kcnmb2, Ccng1 in PDAC module-9 (Fig.3K). Another crosstalk network focuses on the OR superfamily (Fig.3L). GO analysis shows that these genes mainly function through the G-protein coupled receptor (GPCR) signaling pathway. So far, the role of ORs as GPCRs has been underappreciated. Some reports have shown that certain ORs inhibit the proliferation of lung and prostate cancer cell lines[23, 24].

Importantly, we mapped all the significant overlapping and crosstalk genes into human homologies to investigate their expression level in PDAC and their potential relationships with prognosis using the GEPIA tool. We found that SMARCA1, SMARCA2, CLIP3, BPTF and H2AFZ were significantly overexpressed in PDAC compared with normal pancreatic tissue (Fig. 4A). KCNMB2 and H2AFP (human homology of mouse HIST1H2AN), SMARCA1, CLIP3 and SMARCA2 are putative prognostic factors for PDAC (Fig. 4B). Information on the expression and prognosis of other overlapping and crosstalk genes are provided in Fig. S1.

\section{Non-coding RNAs regulating ADM-PanIN-PDAC}

Finally, we predicted the IncRNAs and microRNAs that regulate the overlapping and crosstalk genes during ADM-PanIN-PDAC progression with the miRDB database. There are 44 IncRNAs and 56 microRNAs that regulate the overlapping genes (Fig.5). Statistical information on these ncRNAs is shown in Table S5. As the node degree increases, the relationship between the non-coding RNAs and their related genes becomes more significant. The top five most highly regulated microRNAs were mmu-miR-335-5p, mmu-miR-669n, mmu-miR-7646-5p, mmu-miR-1191b-3p, mmu-miR-1224-5p. These miRNAs target two overlapping genes: Hist1h4m and Hist4h4. The top 10 most highly regulated IncRNAs were C530005A16Rik, 1810062018Rik, 2010204K13Rik, 2810002D19Rik, 4732463B04Rik, H19, Pvt1, Gm14207, Gas5 and Mir17hg. They mainly target the overlapping gene Hist1h2an.

572 microRNAs and 55 IncRNA significantly regulate the crosstalk genes (Table S6). The top 5 microRNAs with greatest node degree are mmu-miR-325-3p, mmu-miR-7661-5p, mmu-miR-590-3p, mmu-miR-743a-3p, mmu-miR-743b-3p. Gm14207,H19, 2610203C20Rik, C130021I20Rik, 1700086L19Rik, 2810002D19Rik, BC006965, D630029K05Rik, Meg3 and A330023F24Rik were the top 10 IncRNAs with greatest node degree (Fig. 6). 2810002D19Rik, H19 and $\mathrm{Gm} 14207$ were predicted to regulate both crosstalk and overlapping genes.

\section{Discussion}

This study unveiled the potential genetic alterations and their regulators that drive ADM to PanIN and subsequently PDAC. We identified 45 genes in the ADM stage, 7 genes in PanIN and 28 genes in PDAC that interact with each other. Overlapping and crosstalk genes in ADM-PanIN-PDAC contain several bridging ADM gene modules (e.g. Hist2h2ab and smarca1), PanIN gene modules (e.g. Hdac11 and smarca2) and PDAC gene modules (e.g. Olfr239 and Hdac6). GO 
analysis shows that these genes mainly play roles in nucleosome assembly, chromatin organization and the G-protein coupled receptor signaling pathway. Our results show that the expression of cancer-related genes is affected by epigenetic dysregulation via DNA methylation and histone modification and by small non-coding regulatory microRNAs (e.g. mmu-miR-335-5p) and IncRNAs (e.g. H19).

Overlapping genes and most crosstalk genes belong to the histone family. The histone is a highly conserved protein with extensive cellular functions. It constitutes two functional parts: core histones (H2A, H2B, H3 and H4) and linker histones ( $\mathrm{H} 1$ and $\mathrm{H} 5)$ [25]. In our study, most overlapping genes are core histones, including HIST1H2AN, also known as H2AC22 (homology of human H2AC11), and three members of Histone H4 (Hist1h4c, Hist1h4m and Hist4h4). Crosstalk genes including H2AFZ (synonym of H2A.Z) were annotated in ADM-PanIN-PDAC. Histones are associated with cancer-predisposing inflammation or even trans-differentiation[26]. Histone $\mathrm{H} 3$ has the potential to be a biomarker for evaluating the severity of acute pancreatitis due to caerulein-triggered extensive pancreatic acinar cell death in animal models[27]. Histone $\mathrm{H} 4$ binds to smooth muscle cells and triggers arterial tissue inflammation[28]. Histone variant $\mathrm{H} 2 \mathrm{~A}$.J accumulates with aging in specific tissues and may contribute to chronic inflammation, aging-associated disease and cancers[29]. $\mathrm{H} 2 \mathrm{AFZ}$ has been reported to be upregulated in breast, liver, bladder and lung cancer and has oncogenic properties in prostate cancer[30]. Our study is the first to demonstrate the potential involvement of H2AFZ in ADM driven PDAC carcinogenesis.

Apart from the differential expression level of histone variants, modification of histones could also exert effects on carcinogenesis[31]. We found several histone deacetylases (HDAC) including HDAC3 in ADM module-1, HDAC11 in PanIN module-4 and HDAC6 in PDAC module-9 that are important in the network of ADM-PanIN-PDAC. High expression of HDAC3 in precursor lesions of prostate cancer has been reported, indicating a critical role in the initiation stage of tumorigenesis[32]. Aberrant expression of HDAC6 also plays critical roles in cell differentiation, apoptosis and cell cycle control[33]. Hdac11 has been identified as a novel target in antitumor therapy[34]. Loss of histone trimethyl transferase, H3K36, facilitates ADM formation through epigenetic dysregulation and leads to extracellular matrix (ECM) production in PDAC[35].

Many genes predicted to function in ADM-PanIN-PDAC regulation were found to interact with histones to different extents. Several genes annotated in our study have been previously validated in PDAC. Absence of ATRX in adult mice with oncogenic KRAS mutation, a subgroup of SWI/SNF complex (Switch/Sucrose Non-Fermentable chromatin remodeling), resulted in increased ADM and progressive PanIN lesions[36]. Deletion of ARID1A, a SWI/SNF component, in the pancreas exaggerated ADM formation, diminished regeneration after injury and lead to intraductal papillary mucinous neoplasm and PanIN when it cooperated with mutant KRAS[37]. We found that another family member, ARID4A, predicted in the ADM module, may also play a role in PDAC initiation. The crosstalk gene, SMARCA1, has been linked with inflammation related diseases and cancer cell proliferation, migration, growth, death and DNA damage[38, 39]. Two other members of the SMARCA class have been successfully linked to PDAC. SMARCA2, annotated in the PanIN module, was correlated with cancer growth, chemoresistance and poor survival of pancreatic cancer patients[40]. SMARCA4-deficient mice in cooperation with oncogenic Kras promoted PDAC precursor lesions[41]. SMARCA1 may act upstream of p53 (a very common mutation in PDAC) by regulating the expression of p53 gene through the Wnt pathway, thus it could represent a prognostic biomarker for PDAC and warrants further research.

Another crosstalk gene, CHD1, is a chromatin remodeling factor which specifically binds to methylated histone $\mathrm{H} 3$ lysine 4 residue (H3K4me3), and is involved in nuclear shuttling in pancreatic cancer cells[42]. Similarly, BPTF (bromodomain PHD transcription factor) could also bind with H3K4me3 to stabilize the NURF (nucleosome remodeling factor) complex on chromatin, resulting in transcriptional regulation[43]. BPTF was expressed at intermediate levels in PDACderived cell lines and is responsible for cancer cell proliferation and PDAC initiation and maintenance by interacting with c-MYC[44]. FRK plays a role in pancreatic cancer cell migration and proliferation, and was suggested to be a critical therapeutic target of pancreatic cancer[45]. CLIP3, a known antiinflammatory regulator involved in TNF-a signaling and injury healing [46],was identified in our study as a novel target of PDAC in the crosstalk gene network. CECR2 functions with SMARCA1 in the CERF complex (CECR2-containing remodeling factor) to regulate cell differentiation and development[47]. Interestingly, SMARCA1, SMARCA2 and CLIP3 were overexpressed in human PDAC and their expression level predicts the patient's overall survival.

We show that olfactory receptor (OR/OLFR) superfamily genes are relatively independent modules involved in PDAC formation. Emerging data has shown that ORs are correlated with cell invasiveness[48] and could function as putative drivers of cancer. Olfactory receptor (OR/OLFR) superfamily genes belong to the $G$ protein-coupled receptor (GPCR) superfamily which are involved in the inflammatory response and cancer development via NF-kB signaling[49]. Our findings on the 22 ORs linked to ADM-PanIN-PDAC support the notion that the olfactory transduction pathway is associated with an elevated risk of pancreatic cancer [50]. Another OR member, prostate-specific G-protein-coupled receptor (PSGR/OR51E2), was upregulated in prostate cancer and could allegedly induce prostatitis at early age in the mouse and promote prostatic intraepithelial neoplasia[51]. MiRNA-374a and miRNA-410, putative regulators of cancer-related genes, were inversely correlated with PSGR overexpression[52]. Recently, overexpression of Olfr544, Olfr543 and Olfr1349 at the mRNA level were found to play a role in regulating glucagon secretion in pancreatic cells[53]. ORs are linked to H2AFZ and the histone superfamily through 4 IncRNAs (Gm9866, 4833422C13Rik, BC016548 and 2810407A14Rik). Exploring the relationship between the spectrum of OR and cancer is invaluable for kindling new therapeutic targets.

It is well known that long noncoding RNAs (IncRNAs) and small noncoding RNAs (miRNA) facilitate tumor initiation and progression through regulating tumor suppressor genes or oncogenes. Our analysis revealed a complex network among miRNAs, IncRNAs and their targeted genes during PDAC tumorigenesis. Consistently, one of our strongest candidates, Gas5, was recently shown to regulate pancreatic cancer metastasis via PTEN[54]. MiR-143, reported to be suppressed in colorectal neoplasia[55], was predicted to regulate ADM-PanIN-PDAC via targeting of ACTR10. MiR-193b, validated as a tumor suppressor in various cancer types, was found to regulate CLIP3 and interact with oncogenic IncRNA (MIR31HG) in our network[56]. Another tumor suppressor Meg3, predicted to interact with Hist1h2bn in our study, was found to be involved in pancreatic cancer[57]. H19, identified as an oncogenic IncRNA, plays a role in pancreatic cancer invasion and metastasis[55]. In line with previous findings, we identified H19 as the top IncRNA in crosstalk with ADM, PanIN and PDAC. miR-335-5p, miR-7646-5p and miR-669n target both Hist4h4 and Hist1h4m. In the literature there is only one study that suggests that MiR-335-5p may attenuate pancreatic cancer development trough modulating the downstream oncogene[58]. The newly identified biological functions of these IncRNA/miRNAs in PDAC remain to be validated in further mechanistic and functional studies. Other tumor suppressors were also annotated in our network.

Page 5/14 
Although it has been well documented in the literature that persistent ADM could progress to PanIN, and finally to invasive PDAC [7, 59-61], PDAC may arise from ADM without the need for PanIN as an intermediate step. The results derived from this study do not apply to those PDAC arising directly from ADM or other precursors such a mucinous cystic neoplasia or intraductal papillary mucinous neoplasm. Furthermore, this bioinformatic analysis is primarily based on animal data, and validation in patient samples and functional studies are required to determine whether these findings will be clinically useful.

\section{Conclusion}

In summary, we revealed potential molecular mechanisms that could regulate the progression of ADM-PanIN-PDAC at both genetic and epigenetic (differentially expressed genes, miRNA and IncRNA) levels. SMARCA1, SMARCA2 and CLIP3 were not only significant crosstalk genes, but also differentially expressed genes and potential prognostic factors for human PDAC. These newly identified genes and their regulators, once validated by further mechanistic and functional studies, could open a new window of opportunity for early detection, prevention, and targeted therapy of PDAC in a clinical setting.

\section{Abbreviations}

PDAC: pancreatic ductal adenocarcinoma.

ADM: acinar ductal metaplasia.

PanIN: pancreatic intraepithelial neoplasia.

SMARCA1: SWI/SNF related, matrix associated, actin dependent regulator of chromatin, subfamily a, member 1.

SMACAR2: SWI/SNF related, matrix associated, actin dependent regulator of chromatin, subfamily a, member 2.

CLIP3: $\quad$ CAP-GLY domain containing linker protein 3

BRAF: Braf transforming gene.

KRAS: Kirsten rat sarcoma viral oncogene homolog.

STRING: the search tool for the retrieval of interacting genes.

PPI: protein-protein interaction.

DAVID: gene expression profiling interactive analysis.

AKT2: thymoma viral proto-oncogene 2 .

MYC: myelocytomatosis oncogene.

CDKN2A: cyclin dependent kinase inhibitor 2A.

TP53: transformation related protein 53.

SMAD4: SMAD family member 4.

BRCA2: breast cancer 2, early onset.

GATA6: GATA binding protein 6 .

MAPK: mitogen-activated protein kinase

PI3K: phosphoinositol-3 kinase.

TGF- $\beta$ : transforming growth factor- $\beta$.

DEG: differentially expressed genes.

OR: olfactory receptor.

Chd1:chromodomain helicase DNA binding protein 1.

Bptf: bromodomain PHD finger transcription factor.

Cecr2: CECR2, histone acetyl-lysine reader.

Frk: fyn-related kinase.

H2afz:H2A.Z variant histone 1.

Kcnmb2:potassium large conductance calcium-activated channel, subfamily M, beta member 2 . 
Ccng1: cyclin G1.

HIST1H2AN: H2A clustered histone 22.

\section{Declarations}

Ethics approval and consent to participate: Not applicable.

Consent for publication囚Not applicable.

Availability of data and material: All data generated or analysed during this study are included in this published article [and its supplementary information files].

Competing interests: The authors declare that they have no competing interests.

Funding: NSFC (Natural Science Foundation of China) (No. 81800573)

Authors' contributions: B.G. analyzed and interpreted data. HR.Z. performed statistical analysis, interpreted the data and was a major contributor in writing the manuscript. BY.S. interpreted the data. ZH.G. analysed the data and revise the manuscript and approved the final manuscript.

Acknowledgements The authors declare no competing interests.

\section{References}

1. Ying H, Dey P, Yao W, Kimmelman AC, Draetta GF, Maitra A, DePinho RA: Genetics and biology of pancreatic ductal adenocarcinoma. Genes Dev 2016, 30(4):355-385.

2. Siegel RL, Miller KD, Jemal A: Cancer statistics, 2019. CA: a cancer journal for clinicians 2019, 69(1):7-34.

3. Eckel F, Brunner T, Jelic S: Biliary cancer: ESMO Clinical Practice Guidelines for diagnosis, treatment and follow-up. Ann Oncol 2011, 22 Suppl 6:vi40-44.

4. Duell EJ, Lucenteforte E, Olson SH, Bracci PM, Li D, Risch HA, Silverman DT, Ji BT, Gallinger S, Holly EA et al: Pancreatitis and pancreatic cancer risk: a pooled analysis in the International Pancreatic Cancer Case-Control Consortium (PanC4). Ann Oncol 2012, 23(11):2964-2970.

5. Guerra C, Schuhmacher AJ, Canamero M, Grippo PJ, Verdaguer L, Perez-Gallego L, Dubus P, Sandgren EP, Barbacid M: Chronic pancreatitis is essential for induction of pancreatic ductal adenocarcinoma by K-Ras oncogenes in adult mice. Cancer cell 2007, 11(3):291-302.

6. Collins MA, Yan W, Sebolt-Leopold JS, Pasca di Magliano M: MAPK signaling is required for dedifferentiation of acinar cells and development of pancreatic intraepithelial neoplasia in mice. Gastroenterology 2014, 146(3):822-834.e827.

7. Zhu L, Shi G, Schmidt CM, Hruban RH, Konieczny SF: Acinar cells contribute to the molecular heterogeneity of pancreatic intraepithelial neoplasia. Am J Pathol 2007, 171(1):263-273.

8. Murtaugh LC, Keefe MD: Regeneration and repair of the exocrine pancreas. Annu Rev Physiol 2015, 77:229-249.

9. Krah NM, De La OJ, Swift GH, Hoang CQ, Willet SG, Chen Pan F, Cash GM, Bronner MP, Wright CV, MacDonald RJ et al: The acinar differentiation determinant PTF1A inhibits initiation of pancreatic ductal adenocarcinoma. Elife 2015, 4.

10. Habbe N, Shi G, Meguid RA, Fendrich V, Esni F, Chen H, Feldmann G, Stoffers DA, Konieczny SF, Leach SD et al: Spontaneous induction of murine pancreatic intraepithelial neoplasia (mPanIN) by acinar cell targeting of oncogenic Kras in adult mice. Proceedings of the National Academy of Sciences of the United States of America 2008, 105(48):18913-18918.

11. Murphy SJ, Hart SN, Lima JF, Kipp BR, Klebig M, Winters JL, Szabo C, Zhang L, Eckloff BW, Petersen GM et al: Genetic alterations associated with progression from pancreatic intraepithelial neoplasia to invasive pancreatic tumor. Gastroenterology 2013, 145(5):1098-1109.e1091.

12. Jones S, Zhang X, Parsons DW, Lin JC, Leary RJ, Angenendt P, Mankoo P, Carter H, Kamiyama H, Jimeno A et al: Core signaling pathways in human pancreatic cancers revealed by global genomic analyses. Science 2008, 321(5897):1801-1806.

13. Integrated Genomic Characterization of Pancreatic Ductal Adenocarcinoma. Cancer cel/ 2017, 32(2):185-203.e113.

14. Rishi A, Goggins M, Wood LD, Hruban RH: Pathological and molecular evaluation of pancreatic neoplasms. Seminars in oncology 2015, 42(1):28-39.

15. Guo M, Jia Y, Yu Z, House MG, Esteller M, Brock MV, Herman JG: Epigenetic changes associated with neoplasms of the exocrine and endocrine pancreas. Discovery medicine 2014, 17(92):67-73.

16. Taucher V, Mangge H, Haybaeck J: Non-coding RNAs in pancreatic cancer: challenges and opportunities for clinical application. Cellular Oncology 2016, 39(4):295-318

17. Reichert M, Takano S, von Burstin J, Kim SB, Lee JS, Ihida-Stansbury K, Hahn C, Heeg S, Schneider G, Rhim AD et al: The Prrx1 homeodomain transcription factor plays a central role in pancreatic regeneration and carcinogenesis. Genes Dev 2013, 27(3):288-300.

18. Schreiber FS, Deramaudt TB, Brunner TB, Boretti MI, Gooch KJ, Stoffers DA, Bernhard EJ, Rustgi AK: Successful growth and characterization of mouse pancreatic ductal cells: functional properties of the Ki-RAS(G12V) oncogene. Gastroenterology 2004, 127(1):250-260.

19. Wettenhall JM, Smyth GK: limmaGUl: a graphical user interface for linear modeling of microarray data. Bioinformatics 2004, 20(18):3705-3706.

20. Gao B, Yu T, Xue D, Sun B, Shao Q, Choudhry H, Marcus V, Ragoussis J, Zhang Y, Zhang W et al: A multidimensional integration analysis reveals potential bridging targets in the process of colorectal cancer liver metastasis. PLoS One 2017, 12(6):e0178760. 
21. Tang Z, Li C, Kang B, Gao G, Li C, Zhang Z: GEPIA: a web server for cancer and normal gene expression profiling and interactive analyses. Nucleic acids research 2017, 45(W1):W98-w102.

22. Jung H, Chae YC, Kim JY, Jeong OS, Kook H, Seo SB: Regulatory role of G9a and LSD1 in the Transcription of Olfactory Receptors during Leukaemia Cell Differentiation. Sci Rep 2017, 7:46182.

23. Kalbe B, Schulz VM, Schlimm M, Philippou S, Jovancevic N, Jansen F, Scholz P, Lubbert H, Jarocki M, Faissner A et al: Helional-induced activation of human olfactory receptor 2J3 promotes apoptosis and inhibits proliferation in a non-small-cell lung cancer cell line. Eur J Cell Bio/ 2017, 96(1):34-46.

24. Neuhaus EM, Zhang W, Gelis L, Deng Y, Noldus J, Hatt H: Activation of an olfactory receptor inhibits proliferation of prostate cancer cells. J Biol Chem 2009, 284(24):16218-16225.

25. Chen R, Kang R, Fan XG, Tang D: Release and activity of histone in diseases. Cell Death Dis 2014, 5(8):e1370-e1370.

26. Kitchen MO, Bryan RT, Emes RD, Glossop JR, Luscombe C, Cheng KK, Zeegers MP, James ND, Devall AJ, Mein CA et al: Quantitative genome-wide methylation analysis of high-grade non-muscle invasive bladder cancer. Epigenetics 2016, 11(3):237-246.

27. Ou X, Cheng Z, Liu T, Tang Z, Huang W, Szatmary P, Zheng S, Sutton R, Toh CH, Zhang N et al: Circulating Histone Levels Reflect Disease Severity in Animal Models of Acute Pancreatitis. Pancreas 2015, 44(7):1089-1095.

28. Silvestre-Roig C, Braster Q, Wichapong K, Lee EY, Teulon JM, Berrebeh N, Winter J, Adrover JM, Santos GS, Froese A et al: Externalized histone H4 orchestrates chronic inflammation by inducing lytic cell death. Nature 2019, 569(7755):236-240.

29. Contrepois K, Coudereau C, Benayoun BA, Schuler N, Roux P-F, Bischof O, Courbeyrette R, Carvalho C, Thuret J-Y, Ma Z et al: Histone variant H2A.J accumulates in senescent cells and promotes inflammatory gene expression. Nat Commun 2017, 8:14995-14995.

30. Vardabasso C, Hasson D, Ratnakumar K, Chung C-Y, Duarte LF, Bernstein E: Histone variants: emerging players in cancer biology. Cell Mol Life Sci 2014, 71(3):379-404

31. Audia JE, Campbell RM: Histone Modifications and Cancer. Cold Spring Harb Perspect Bio/ 2016, 8(4):a019521-a019521.

32. Weichert W, Röske A, Gekeler V, Beckers T, Stephan C, Jung K, Fritzsche FR, Niesporek S, Denkert C, Dietel M et al: Histone deacetylases 1, 2 and 3 are highly expressed in prostate cancer and HDAC2 expression is associated with shorter PSA relapse time after radical prostatectomy. British Journal of Cancer 2008, 98(3):604-610.

33. Mair B, Kubicek S, Nijman SM: Exploiting epigenetic vulnerabilities for cancer therapeutics. Trends Pharmacol Sci 2014, 35(3):136-145.

34. Deubzer HE, Schier MC, Oehme I, Lodrini M, Haendler B, Sommer A, Witt O: HDAC11 is a novel drug target in carcinomas. Int J Cancer 2013, 132(9):22002208.

35. Niu N, Lu P, Yang Y, He R, Zhang L, Shi J, Wu J, Yang M, Zhang ZG, Wang LW et al: Loss of Setd2 promotes Kras-induced acinar-to-ductal metaplasia and epithelia-mesenchymal transition during pancreatic carcinogenesis. Gut 2020, 69(4):715-726.

36. Young CC, Baker RM, Howlett CJ, Hryciw T, Herman JE, Higgs D, Gibbons R, Crawford H, Brown A, Pin CL: The Loss of ATRX Increases Susceptibility to Pancreatic Injury and Oncogenic KRAS in Female But Not Male Mice. Cell Mol Gastroenterol Hepatol 2018, 7(1):93-113.

37. Wang W, Friedland SC, Guo B, O'Dell MR, Alexander WB, Whitney-Miller CL, Agostini-Vulaj D, Huber AR, Myers JR, Ashton JM et al: ARID1A, a SWI/SNF subunit, is critical to acinar cell homeostasis and regeneration and is a barrier to transformation and epithelial-mesenchymal transition in the pancreas. Gut 2019, 68(7):1245-1258.

38. Ye Y, Xiao Y, Wang W, Wang Q, Yearsley K, Wani AA, Yan Q, Gao J-X, Shetuni BS, Barsky SH: Inhibition of Expression of the Chromatin Remodeling Gene, \&lt;em\&gt;SNF2L\&lt;/em\&gt; Selectively Leads to DNA Damage, Growth Inhibition, and Cancer Cell Death. Molecular Cancer Research 2009, 7(12):1984.

39. Eckey M, Kuphal S, Straub T, Rummele P, Kremmer E, Bosserhoff AK, Becker PB: Nucleosome remodeler SNF2L suppresses cell proliferation and migration and attenuates Wnt signaling. Molecular and cellular biology 2012, 32(13):2359-2371.

40. Zhang Z, Wang F, Du C, Guo H, Ma L, Liu X, Kornmann M, Tian X, Yang Y: BRM/SMARCA2 promotes the proliferation and chemoresistance of pancreatic cancer cells by targeting JAK2/STAT3 signaling. Cancer Letters 2017, 402:213-224.

41. von Figura G, Fukuda A, Roy N, Liku ME, Morris Iv JP, Kim GE, Russ HA, Firpo MA, Mulvihill SJ, Dawson DW et al: The chromatin regulator Brg1 suppresses formation of intraductal papillary mucinous neoplasm and pancreatic ductal adenocarcinoma. Nat Cell Biol 2014, 16(3):255-267.

42. Dey P, Ponnusamy MP, Deb S, Batra SK: Human RNA polymerase ll-association factor 1 (hPaf1/PD2) regulates histone methylation and chromatin remodeling in pancreatic cancer. PLoS One 2011, 6(10):e26926-e26926.

43. Li H, Ilin S, Wang W, Duncan EM, Wysocka J, Allis CD, Patel DJ: Molecular basis for site-specific read-out of histone H3K4me3 by the BPTF PHD finger of NURF. Nature 2006, 442(7098):91-95.

44. Richart L, Carrillo-de Santa Pau E, Río-Machín A, de Andrés MP, Cigudosa JC, Lobo VJS-A, Real FX: BPTF is required for c-MYC transcriptional activity and in vivo tumorigenesis. Nat Commun 2016, 7:10153-10153.

45. Je DW, O YM, Ji YG, Cho Y, Lee DH: The inhibition of SRC family kinase suppresses pancreatic cancer cell proliferation, migration, and invasion. Pancreas 2014, 43(5):768-776.

46. Chiricosta L, Silvestro S, Pizzicannella J, Diomede F, Bramanti P, Trubiani O, Mazzon E: Transcriptomic Analysis of Stem Cells Treated with Moringin or Cannabidiol: Analogies and Differences in Inflammation Pathways. International journal of molecular sciences 2019, 20(23).

47. Banting GS, Barak O, Ames TM, Burnham AC, Kardel MD, Cooch NS, Davidson CE, Godbout R, McDermid HE, Shiekhattar R: CECR2, a protein involved in neurulation, forms a novel chromatin remodeling complex with SNF2L. Human molecular genetics 2005, 14(4):513-524.

48. Sanz G, Leray I, Dewaele A, Sobilo J, Lerondel S, Bouet S, Grebert D, Monnerie R, Pajot-Augy E, Mir LM: Promotion of cancer cell invasiveness and metastasis emergence caused by olfactory receptor stimulation. PLoS One 2014, 9(1):e85110.

Page 8/14 
49. Stender JD, Pascual G, Liu W, Kaikkonen MU, Do K, Spann NJ, Boutros M, Perrimon N, Rosenfeld MG, Glass CK: Control of proinflammatory gene programs by regulated trimethylation and demethylation of histone H4K20. Mol Cell 2012, 48(1):28-38.

50. Paladino D, Yue P, Furuya H, Acoba J, Rosser CJ, Turkson J: A novel nuclear Src and p300 signaling axis controls migratory and invasive behavior in pancreatic cancer. Oncotarget 2016, 7(6):7253-7267.

51. Mourtada-Maarabouni M, Pickard MR, Hedge VL, Farzaneh F, Williams GT: GAS5, a non-protein-coding RNA, controls apoptosis and is downregulated in breast cancer. Oncogene 2009, 28(2):195-208.

52. Gao Z-Q, Wang J-f, Chen D-H, Ma X-S, Wu Y, Tang Z, Dang X-W: Long non-coding RNA GAS5 suppresses pancreatic cancer metastasis through modulating miR-32-5p/PTEN axis. Cell \& Bioscience 2017, 7:66.

53. Michael MZ, SM OC, van Holst Pellekaan NG, Young GP, James RJ: Reduced accumulation of specific microRNAs in colorectal neoplasia. Mol Cancer Res 2003, 1(12):882-891.

54. Ma C, Nong K, Zhu H, Wang W, Huang X, Yuan Z, Ai K: H19 promotes pancreatic cancer metastasis by derepressing let-7's suppression on its target HMGA2-mediated EMT. Tumour Biol 2014, 35(9):9163-9169.

55. Yoshimura H, Matsuda Y, Yamamoto M, Michishita M, Takahashi K, Sasaki N, Ishikawa N, Aida J, Takubo K, Arai T et al: Reduced expression of the H19 long non-coding RNA inhibits pancreatic cancer metastasis. Lab Invest 2018, 98(6):814-824.

56. Yang H, Liu P, Zhang J, Peng X, Lu Z, Yu S, Meng Y, Tong WM, Chen J: Long noncoding RNA MIR31HG exhibits oncogenic property in pancreatic ductal adenocarcinoma and is negatively regulated by miR-193b. Oncogene 2016, 35(28):3647-3657.

57. Ma L, Wang F, Du C, Zhang Z, Guo H, Xie X, Gao H, Zhuang Y, Kornmann M, Gao H et al: Long non-coding RNA MEG3 functions as a tumour suppressor and has prognostic predictive value in human pancreatic cancer. Oncol Rep 2018, 39(3):1132-1140.

58. An Y, Cai H, Zhang Y, Liu S, Duan Y, Sun D, Chen X, He X: circZMYM2 Competed Endogenously with miR-335-5p to Regulate JMJD2C in Pancreatic Cancer. Cellular Physiology and Biochemistry 2018, 51(5):2224-2236.

59. Maitra A, Fukushima N, Takaori K, Hruban RH: Precursors to invasive pancreatic cancer. Advances in anatomic pathology 2005, 12(2):81-91.

60. Morris JPt, Cano DA, Sekine S, Wang SC, Hebrok M: Beta-catenin blocks Kras-dependent reprogramming of acini into pancreatic cancer precursor lesions in mice. The Journal of clinical investigation 2010, 120(2):508-520.

61. Storz P: Acinar cell plasticity and development of pancreatic ductal adenocarcinoma. Nature reviews Gastroenterology \& hepatology 2017, 14(5):296-304.

\section{Table}

Table 1. The function of the significant overlapping modules.

Module Genes

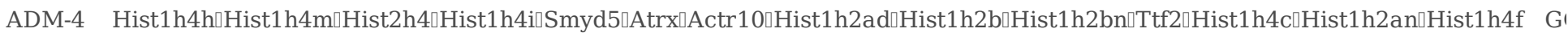

PanIN- Hist1h2an, Hist1h4c, Hist4h4, Hist1h4m, Hist1h2ag, Hist1h2ae, Hist1h4j, Hist3h2a, Hist2h2ac, Top1mt, Hist1h2af, Rbm43, Gı

$1 \quad$ Ercc6 


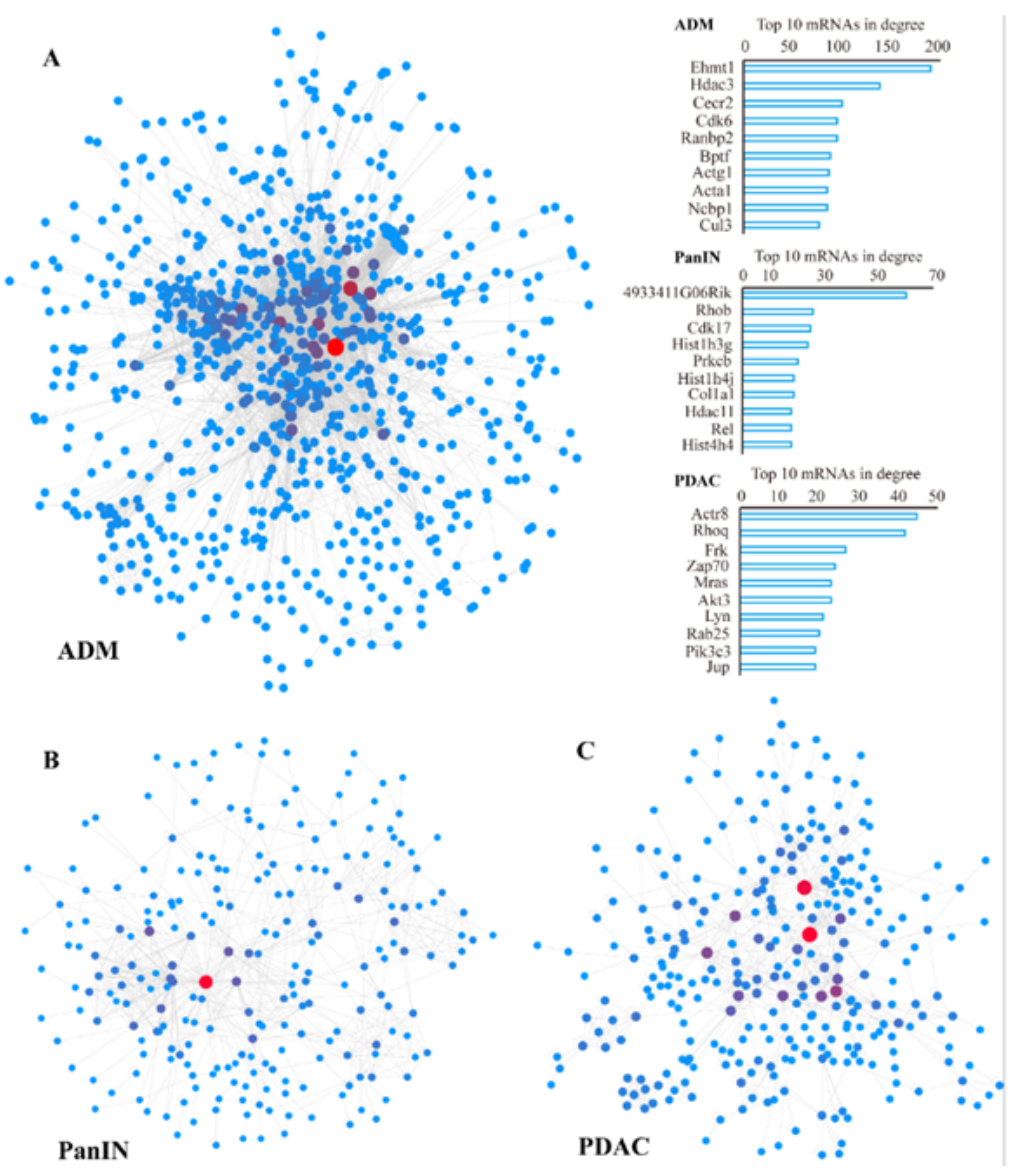

Figure 1

PPI network of differentially expressed genes in ADM (A), PanIN (B) and PDAC (D). The the top 10 mRNAs in degree are illustrated.

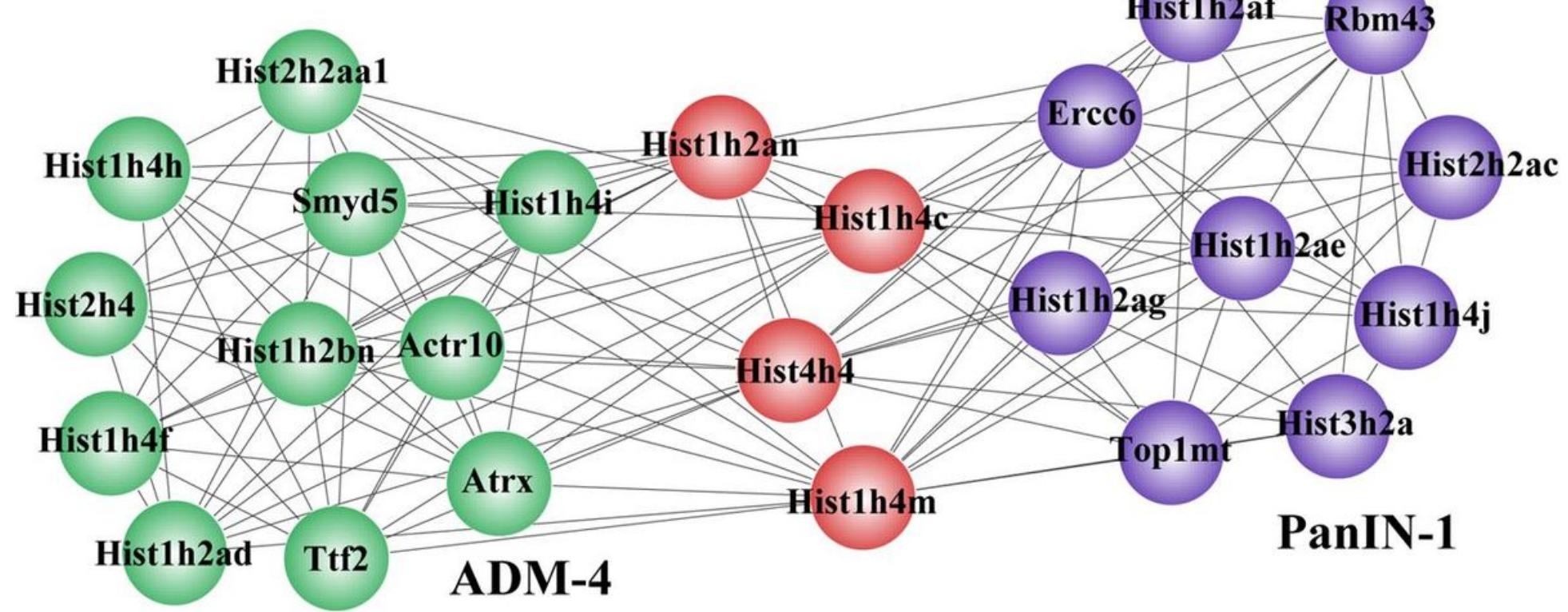

\section{ADM module genes $O$ overlap genes $O$ PanIN module genes}

Figure 2

The overlapping subnetwork of ADM-4 and PanIN-1 modules. Green nodes, purple nodes and red nodes represent the genes in PanIN-1, ADM-4, and the overlapping genes among modules, respectively. Black lines represent gene-gene interactions. 

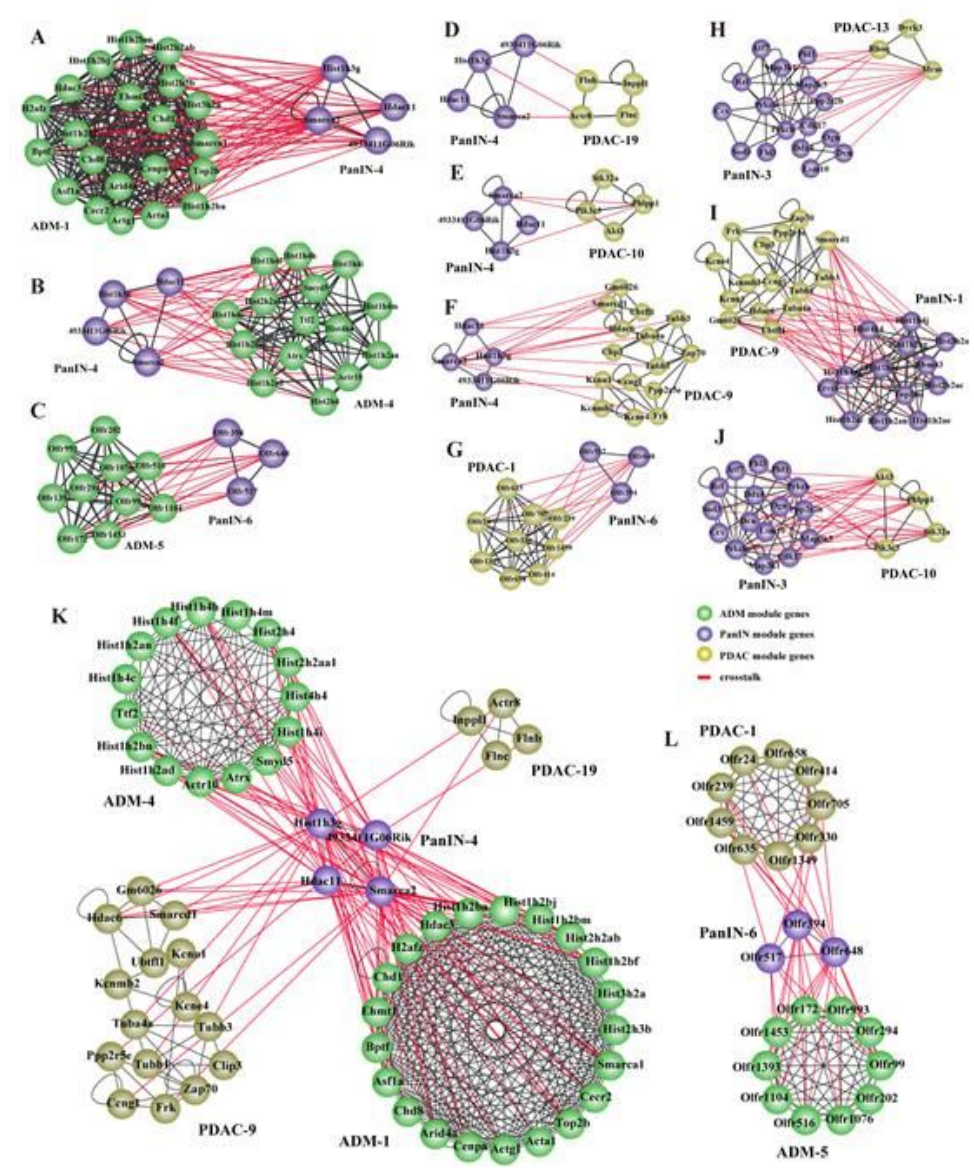

Figure 3

The crosstalk genes in ADM-PanIN-PDAC. (A) The crosstalk subnetwork of ADM-1 and PanIN-4. (B) The crosstalk subnetwork of ADM-4 and PanIN-4. (C) The crosstalk subnetwork of ADM-5 and PanIN-6. (D) The crosstalk subnetwork of PanIN-4 and PDAC-19. (E) The crosstalk subnetwork of PanIN-4 and PDAC-10. (F) The crosstalk subnetwork of PanIN-4 and PDAC-9. (G) The crosstalk subnetwork of PanIN-6 and PDAC-1. (H) The crosstalk subnetwork of PanIN-3 and PDAC-13. (I) The crosstalk subnetwork of PanIN-1 and PDAC-9. (J) The crosstalk subnetwork of PanIN-3 and PDAC-10. (K and L) The combined crosstalk subnetwork of ADM-PanIN-PDAC. Green and purple nodes represent the DEGs in the ADM and PanIN modules, respectively. Red edges represent gene crosstalk. Black edges represent gene interactions in the same module. 


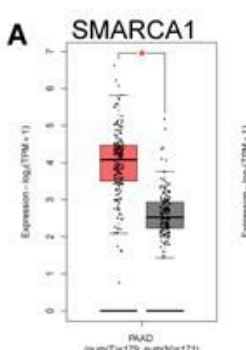

SMARCA2
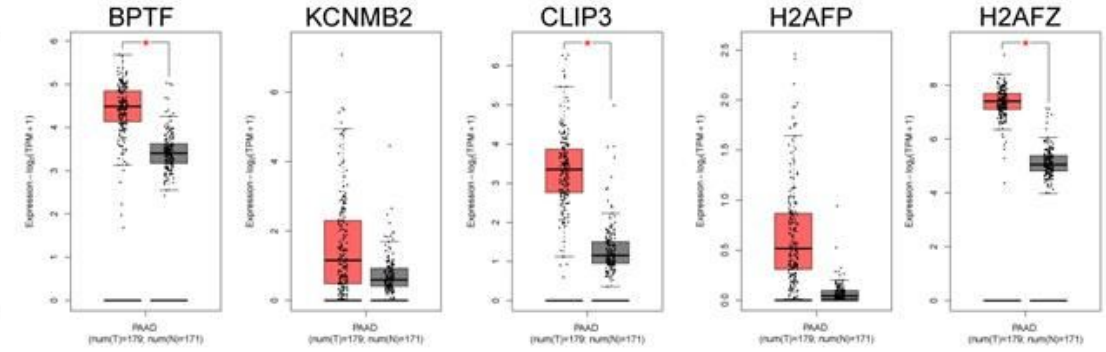

B
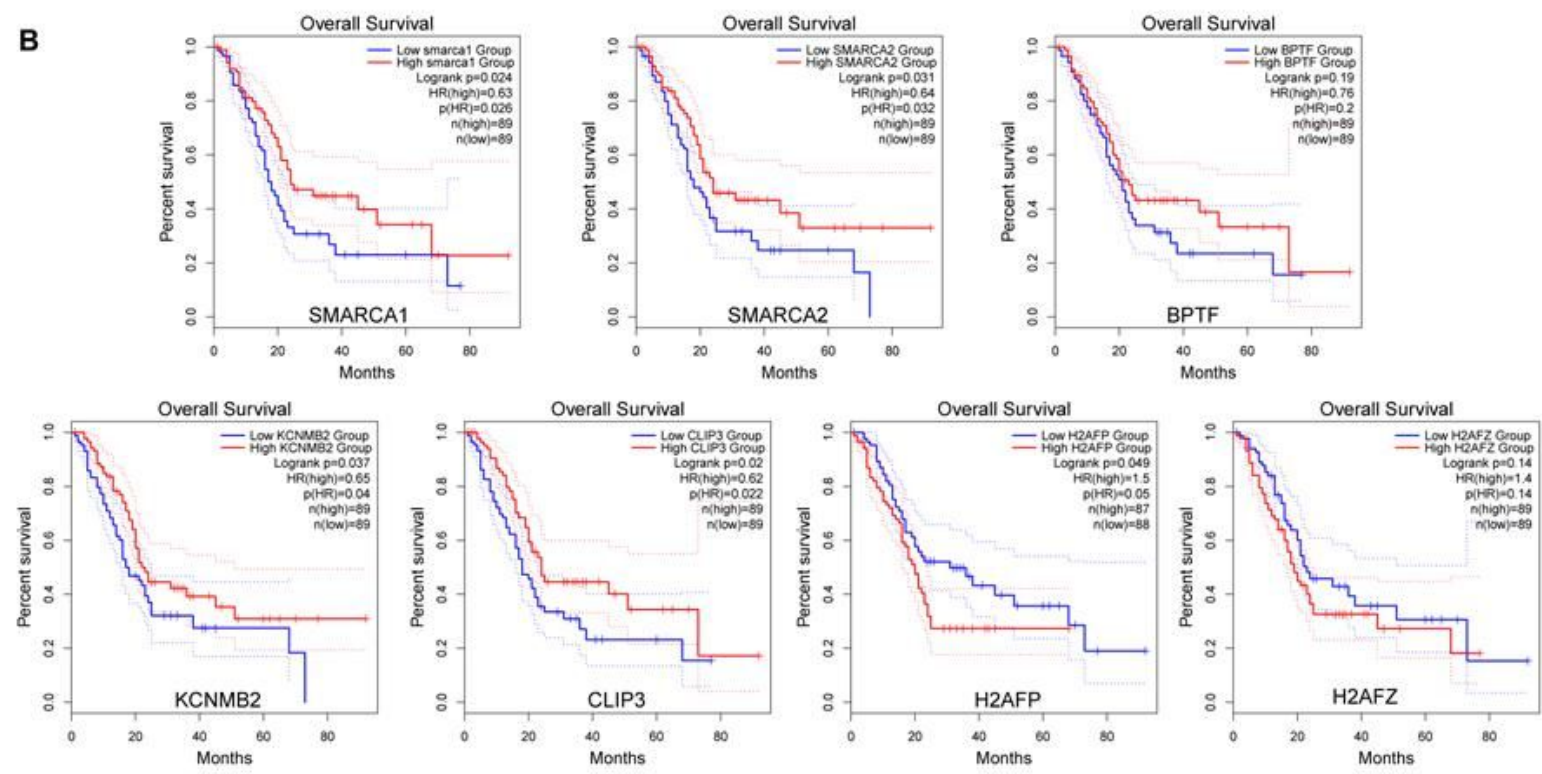

Figure 4

The crosstalk genes in ADM-PanIN-PDAC. (A) The crosstalk subnetwork of ADM-1 and PanIN-4. (B) The crosstalk subnetwork of ADM-4 and PanIN-4. (C) The crosstalk subnetwork of ADM-5 and PanIN-6. (D) The crosstalk subnetwork of PanIN-4 and PDAC-19. (E) The crosstalk subnetwork of PanIN-4 and PDAC-10. (F) The crosstalk subnetwork of PanIN-4 and PDAC-9. (G) The crosstalk subnetwork of PanIN-6 and PDAC-1. (H) The crosstalk subnetwork of PanIN-3 and PDAC-13. (I) The crosstalk subnetwork of PanIN-1 and PDAC-9. (J) The crosstalk subnetwork of PanIN-3 and PDAC-10. (K and L) The combined crosstalk subnetwork of ADM-PanIN-PDAC. Green and purple nodes represent the DEGs in the ADM and PanIN modules, respectively. Red edges represent gene crosstalk. Black edges represent gene interactions in the same module. 


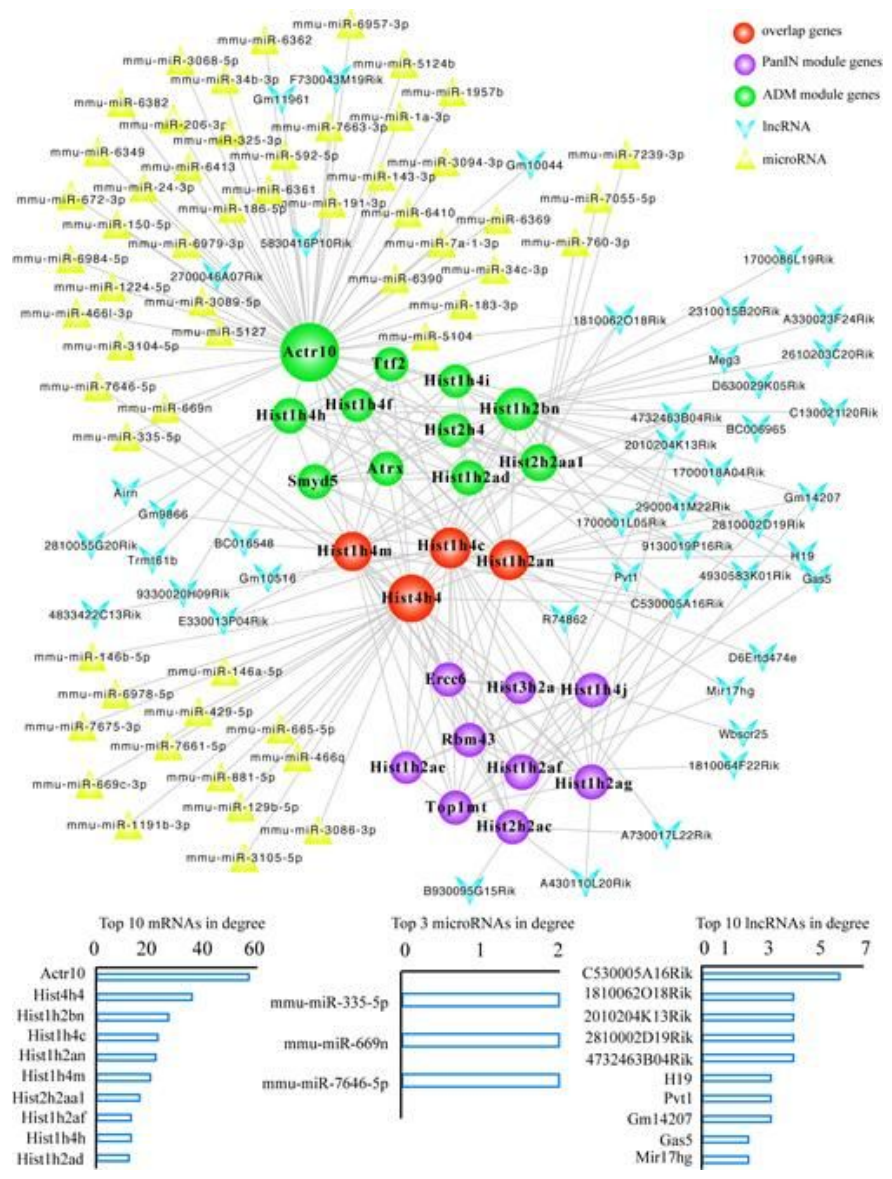

\section{Figure 5}

The microRNA and IncRNA regulatory network of the overlapping genes in ADM and PanIN. Green nodes and purple nodes represent the DEGs in ADM and PanIN, respectively. Red nodes represent the overlap genes. Yellow square nodes and blue rhombus nodes represent microRNAs and IncRNAs targeting the overlapping genes, respectively. 

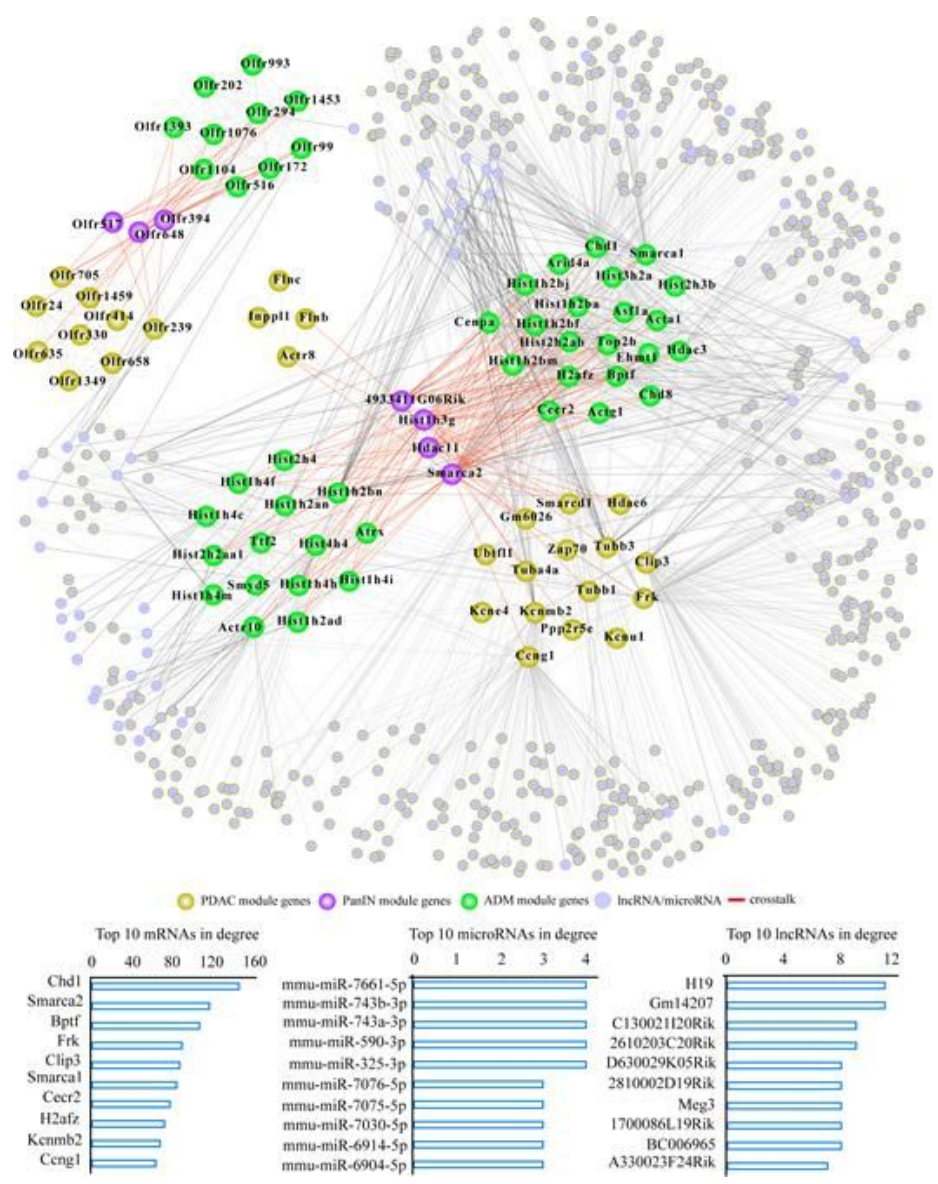

\section{Figure 6}

The significant crosstalk genes in ADM-PanIN-PDAC progression. Green nodes, purple nodes and yellow nodes represent the DEGs in ADM, PanIN and PDAC, respectively. Red lines link the crosstalk genes. Grey nodes represent microRNA and IncRNA.

\section{Supplementary Files}

This is a list of supplementary files associated with this preprint. Click to download.

- Tables1ADMDEGs.xlsx

- TableS2PanINDEGs.xlsx

- Tables3PDACDEGs.xIsx

- Tables4.docx

- Tables5.docx

- Tables6.docx

- Figures1.tif

- Figures2.tif 